\title{
Energy saving in optical transport networks exploiting transmission properties and wavelength path optimization
}

\author{
Antonio Silvestri ${ }^{a}$, Alessandro Valenti ${ }^{a, *}$, Sergio Pompei ${ }^{a}$, Francesco Matera ${ }^{a}$, \\ Antonio Cianfrani ${ }^{\mathrm{b}}$, Angelo Coiro ${ }^{\mathrm{b}}$ \\ a Fondazione Ugo Bordoni, via B. Castiglione 59, 00142 Rome, Italy \\ b University of Rome “La Sapienza", INFOCOM Dept, via Cavour 256, 00184 Rome, Italy
}

\section{A R T I C L E I N F O}

\section{Article history:}

Received 29 July 2009

Received in revised form 23 March 2010

Accepted 12 April 2010

Available online 20 April 2010

\section{Keywords:}

WDM

Transmission performance

Energy saving

Dispersion management

\begin{abstract}
A B S T R A C T
Energy consumption is becoming a fundamental topic also for telecommunication networks, where the implementation of "green" infrastructures is required. In this paper, we report a numerical investigation about energy saving in a transport network both exploiting the transmission properties that permit to reduce the number of in-line equipments (optimizing wavelength path assignment) and switching off optical links when the traffic load decreases. In particular, we investigate a European transport network based on $N \times$ $40 \mathrm{Gbit} / \mathrm{s}$ transmission and the Italian network based on the $M \times 10 \mathrm{Gbit} / \mathrm{s}$ transmission.
\end{abstract}

(C) 2010 Elsevier B.V. All rights reserved.

\section{Introduction}

Today energy saving is becoming a key issue in any economy sector; in particular, the impact of ICT technologies on power consumption is not negligible and, consequently, the implementation of network infrastructures that limit energy consumption is currently a fundamental task. To give an idea, a telecommunication incumbent can reach an energy consumption of some TWh/year [1].

It is clear that power consumption is a crucial topic especially for the implementation of novel network segments that have to be built in the coming years; in particular, it is important for the Next Generation Access Networks that require the installation of very wide bandwidth user connections, and, as a consequence, a deep optical fibre penetration in the access. For example, in [2] it is shown how the introduction of the optical fibre in the access network results in a higher energy saving than radio and copper solutions.

\footnotetext{
* Corresponding author.

E-mail address: avalenti@fub.it (A. Valenti).
}

Conversely, in the coming years, transport networks will not see many investments in terms of fibre deployments and will increase their transmission capacities in terms of higher bit rate optical transmission systems. Therefore, in transport networks energy saving could be achieved by introducing suitable traffic engineering procedures for traffic grooming, with the aim to reduce the number of active links, especially during low traffic periods. Furthermore, some techniques could be adopted with the aim of increasing the transmission capacity but limiting the use of high energy consuming apparatus.

In this work, we investigate on the optimization of transmission elements in a transport network to save Energy, taking into account two aspects: first of all, we exploit the transmission capacity of optical fibre systems to reduce the use of some devices as $3 R$ regenerators [3]; then, we analyze the traffic distribution during the day in order to switch off some fibre links in periods with low load.

In order to better exploit the already installed cables, we assume the use of standard G. 652 fibres, adopting the dispersion management method [4] to achieve Intensity Modulation-Direct Detection (IM-DD) transmission at $10 \mathrm{Gbit} / \mathrm{s}$ and $40 \mathrm{Gbit} / \mathrm{s}$, on long and very long distances. 


\section{Numerical method}

This work has been carried out using the interplay between two simulation tools: a transmission simulation code based on the split-step method [3] to evaluate system performances, and the OPNET ${ }^{\mathrm{TM}}$ (OPtimum NETwork performance) SP Guru Transport Planner tool (version 12.0) [5] to set paths on the network.

The transmission line is supposed to be composed by optical fibres, optical amplifiers and chromatic dispersion compensators based on fibre gratings. We have used an ideal transmitter with return to zero Gaussian pulses. Moreover, in order to better exploit the already installed cables, we assume the use of standard G. 652 fibres, adopting the dispersion management method [4] to achieve IM-DD transmission at $10 \mathrm{Gbit} / \mathrm{s}$ and $40 \mathrm{Gbit} / \mathrm{s}$, on long and very long distances.

The core of the simulation code for transmission performance evaluation is a routine regarding the optical fibre based on the numerical split-step method to solve the nonlinear Schroedinger equation describing the signal propagation in optical fibre [6]. The Erbium Doped Fibre Amplifier (EDFA) is simulated by multiplying the electrical field for the total gain $G$, and by adding to each spectral component of the signal an independent noise term. The real and imaginary parts of the noise spectral components are independent Gaussian variables with variance $[3,6] \sigma^{2}=n_{s p} \mathrm{~h} v(G-1) \Delta v / 2$, where $n_{s p}$ accounts for incomplete population inversion, $h$ is the Plank constant, $v$ is the signal carrier frequency and $\Delta v$ is the bandwidth occupied by each Fourier component of the discrete Fourier spectrum.

Our IM-DD receiver consists of a PIN photodiode and an electrical front end, simulated by a square law device and a Gaussian noise source for the received noise. After detection, the signal is filtered by an electrical filter (simulated with G.957, Bessel-Thomson, filter) with a bandwidth equal to $0.8 R$, where $R$ is the signal bit rate. In front of the receiver, an optical third order Butterworth filter, with a bandwidth equal to $4 R$, is located. The performances are evaluated by means of the $Q$ factor. Our investigation is based on the research of the maximum propagation distance that allows the signal to have the high performance, defined in [3], in terms of $Q$ factor and input power. In particular, such high performance, which permits to have a safe operation condition for the system, is satisfied when we can achieve a power interval $\left[P_{\min }-\right.$ $\left.P_{\max }\right]$, with $P_{\max } / P_{\min }>3$, in which $Q$ is always higher than 7.2 (corresponding to a BER lower than $10^{-12}$ ).

Concerning network operations, OPNET code allows to design optical transport networks. In particular, starting from a traffic matrix and from a physical network topology, OPNET permits to set a connection for each traffic request, solving the Routing and Wavelength Assignment problem and performing the traffic grooming; moreover, by means of OPNET we are also able to establish protection paths, assuming $1+1$ Disjoint Path scheme.

We consider two different network scenarios. In the first case we assume a SONET/SDH transport network with Wavelength Division Multiplexing (WDM) transmission. Here Optical Cross Connects (OXCs) are not present and we refer to this scenario as Opaque Network scenario. Each node consists of Wavelength Division Multiplexing (WDM) TX-RX apparatus with a Digital Cross Connect (DXC) that permits grooming (link by link procedure) of the traffic contributions coming from adjacent nodes; lightpaths cannot cross the nodes and are terminated at each intermediate node. Each lightpath can reach the final destination if its length is compatible with the transmission impairments of the link; otherwise, it has to be in-line $3 R$ regenerated.

The second considered network scenario consists of a SONET/SDH network overlaid to a transparent optical network (we refer to this as Translucent Network scenario). In this case each node consists of a DXC, performing traffic grooming, plus an OXC that permits to set direct lightpaths between no-adjacent nodes: if two no-adjacent nodes require to exchange a traffic higher than $0.5 R$, where $R$ is the channel bit rate, and if the length of the lightpath is compatible with the transmission impairments of links composing the path, a direct lightpath can be assigned to this couple of nodes. In this case, the grooming procedure and wavelength assignment can be evaluated according to the presence of these direct lightpaths. In particular, for direct lightpaths the wavelength assignment algorithm used by OPNET is the First Fit algorithm.

Therefore, in both scenarios, for each traffic request the simulation tool looks for the shortest path accounting the number of hops; if more paths have the same number of hops, the algorithm chooses the one with shortest physical length. Moreover, each traffic demand is considered in the order in which it appears in the traffic matrix.

OPNET tool allows to achieve the complete network design in terms of optical fibre links, optical amplifiers, wavelength channels (including protection paths) and $3 R$ regenerators. The channel bit rate, the amplifiers and the in-line $3 R$ spacing are input data for the OPNET simulation tool; we assign such data by preliminary simulations carried out by the transmission simulation tool. In this work, we only consider WDM RZ IM-DD systems in dispersion management links (i.e., with periodical dispersion compensation) with and without pre-chirp. We assume G.652 fibre links, with chromatic dispersion periodically compensated by means of fibre gratings.

\section{Investigation on the European transport network}

We consider the European transport network with a traffic matrix reported in Fig. 1 and with the fibre links shown in Fig. 2.

We assume the following links parameters: chromatic dispersion equal to $16 \mathrm{ps} / \mathrm{nm} / \mathrm{km}$ (G.652 fibres), nonlinear Kerr effect equal to $1.3 \mathrm{~W} \mathrm{~km}{ }^{-1}$, EDFAs, with $n_{s p}=2$, located at maximum every $100 \mathrm{~km}$, and channel bit rate equal to $40 \mathrm{Gbits} / \mathrm{s}$. Chromatic dispersion is totally compensated by fibre grating, supposed with a loss of $1.5 \mathrm{~dB}$.

In these conditions, the propagation is difficult for long distances: several solutions have been proposed, based either on the chromatic dispersion compensation (dispersion management) or on the use of systems with multilevel modulation formats [7] operating on phase and polarization, without adopting chromatic dispersion 


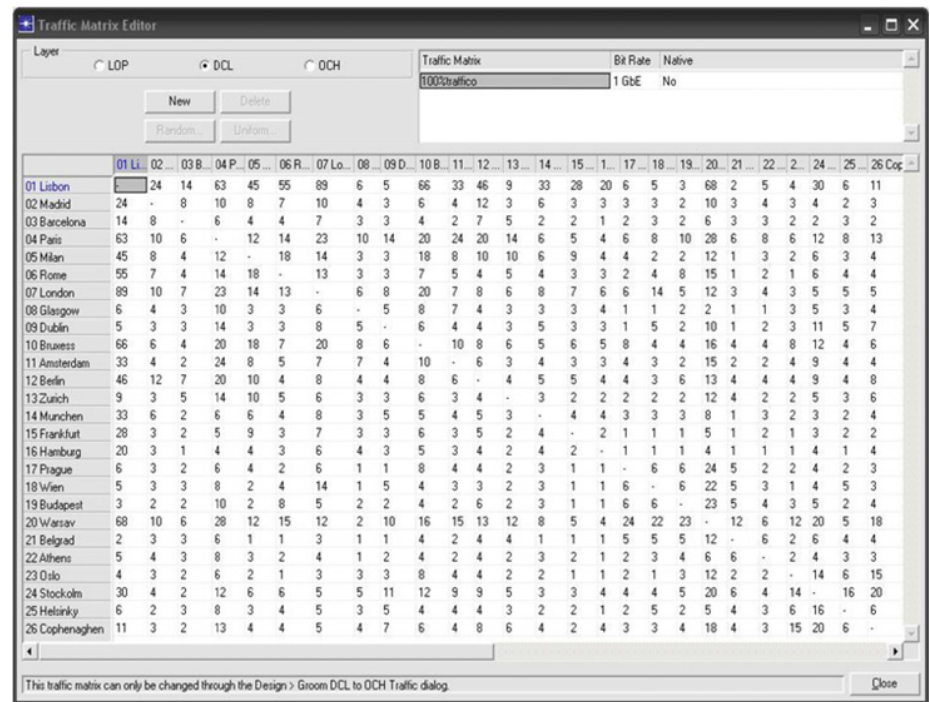

Fig. 1. Traffic matrix.

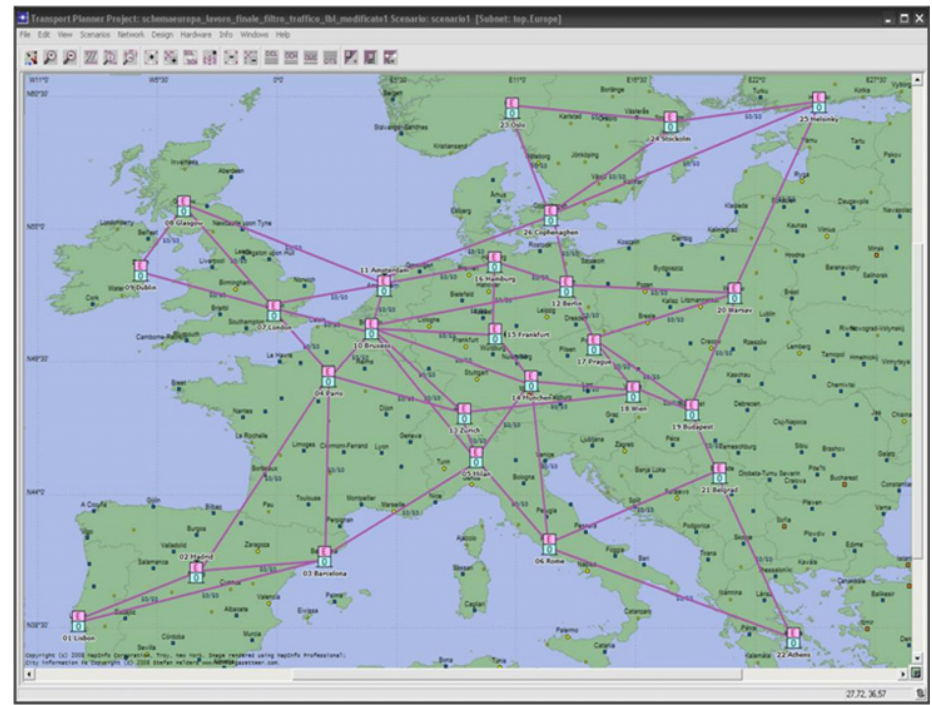

Fig. 2. European network topology.

compensation along the line. For an instance, by adopting dispersion management, in [3] RZ IM-DD systems with the $100 \%$ chromatic dispersion compensation at each amplifier location were proposed (Fig. 3), and it was shown that, without looking at the best pulse duration, the maximum propagation distance is limited to $500 \mathrm{~km}$. However, according to Non Return Zero (NRZ) encoding, for the network under study the distance is limited to $380 \mathrm{~km}$ $(100+100+100+80 \mathrm{~km})$. Therefore, we assumed $380 \mathrm{~km}$ as maximum $3 R$ spacing, that means that without transmission optimization a wide number of $3 R$ devices is required, with consequent huge costs and high energy consumption.

To limit the deployment of $3 R$ devices, we can try to optimize transmission performances in each fibre link by adopting a suitable pulse duration. For an instance, by using RZ and by optimizing the pulse duration (pulses shorter than $7 \mathrm{ps)}$ the maximum propagation distance can reach $1000 \mathrm{~km}$. Furthermore, by locating a small prechirp at link input, the maximum propagation distance for single channel transmission can reach $1300 \mathrm{~km}$ for a pulse duration of $5 \mathrm{ps}$ [3]. On other hand, in the presence of many WDM channels, the maximum propagation distance is limited by the Kerr nonlinearity interplay among the channels, which can be limited by using wider pulses. Therefore, for each fibre link a suitable solution has to be found looking at the link length and at the channel number. It has to be pointed out that both for single channel and WDM transmission, a performance improvement could be achieved by using Raman amplification [3].

In Fig. 3, we report the scheme of the RZ IM-DD system, with and without pre-chirp.

The latter aspect that we have considered is the traffic redistribution in periods of low load (e. g., during night), 


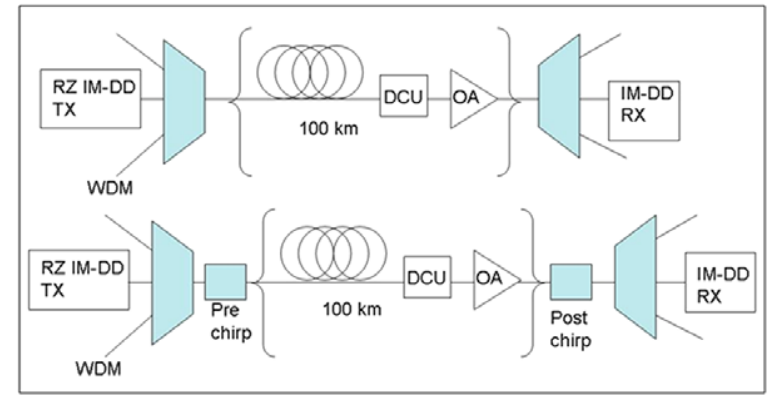

Fig. 3. Scheme of a WDM RZ IM-DD dispersion management link without pre-chirp (up) and with pre-chirp (down). DCU is Dispersion Compensating Unit, OA is Optical Amplifier.

trying to saturate the links carrying higher traffic load and switching off the links with less traffic load. Therefore, first we designed the wavelength distribution in the links in the condition of maximum traffic (Fig. 1), and then, when the traffic decreases, we start with a step-by-step procedure of switching off the links with less load. In particular, links are ordered in increasing values of traffic load and are selected to be eventually switched off starting from the one with minimum load. At each step, traffic flowing across the selected link is re-routed on the remaining links. If an available wavelength can be found for every traffic flow, then the link is considered off and the next link is selected, otherwise algorithm ends. In this procedure, we assume to maintain the same wavelengths in each link, and to keep Disjoint Path protection scheme.

\subsection{Results based on the optimization of the transmission performance}

OPNET Transport Planner, as described in Section 2, allows the traffic grooming in wavelength channels and allows designing link characteristics in terms of fibres, optical amplifiers, DXCs, OXCs and wavelength channels.
Furthermore, without transmission optimization the $3 R$ regeneration is supposed, at maximum, every $380 \mathrm{~km}$. According to these inputs and in Opaque Network scenario, the network dimensioning evaluated by OPNET, requires 50 fibre links with 428 wavelength channels, 194 optical amplifiers and $3683 R$ regenerators. In Table 1 , we report the detail of the number of channels for each link. For sake of space, we do not report the table relative to the restoration paths, consisting of other 50 fibre links.

As example, in Fig. 4 we report the working path and protection path between two nodes (Lisbon-Helsinki) elaborated by means of OPNET.

Looking at the link details, in terms of worse propagation conditions the most critical links are Barcelona-Lisbon (1008 km, 19 channels) and Milan-Barcelona $(725 \mathrm{~km}$, 24 channels): therefore, we can avoid deployment of inline $3 R$ devices by adopting the dispersion management without pre-chirp and by using pulses with a duration equal to 6 ps. In Fig. 5(a), we show the eye diagram at the Barcelona-Lisbon link output (10th Channel). In such a case, the $Q$ factor resulted higher than 7.2 in a wide average power interval (3,5-11 mW). In Fig. 5(b), we report the spectrum at the output of the Milan-Barcelona link.

In Fig. 6, we compare the number of optical amplifiers and $3 R$ apparatus, with and without transmission optimization. In the case of the optimization, we can see an increase of optical amplifiers but zero $3 R$ elements that means a reduction both in terms of costs, since optical amplifiers are cheaper than $3 R$, and in terms of power consumption that we estimate around $1000-2000 \mathrm{~kW}$, assuming a consumption of $200-500 \mathrm{~W}$ for optical amplifier and $2-5 \mathrm{~kW}$ for $3 R$.

We evaluated the wavelength assignment also for Translucent Network scenario, even though for the traffic matrix of Fig. 1 the number of wavelengths crossing the nodes was limited.

In particular, 32 direct lightpaths would be necessary, even though, due to the long distance, most of them required the presence of $3 R$ regenerators, and thus, for these

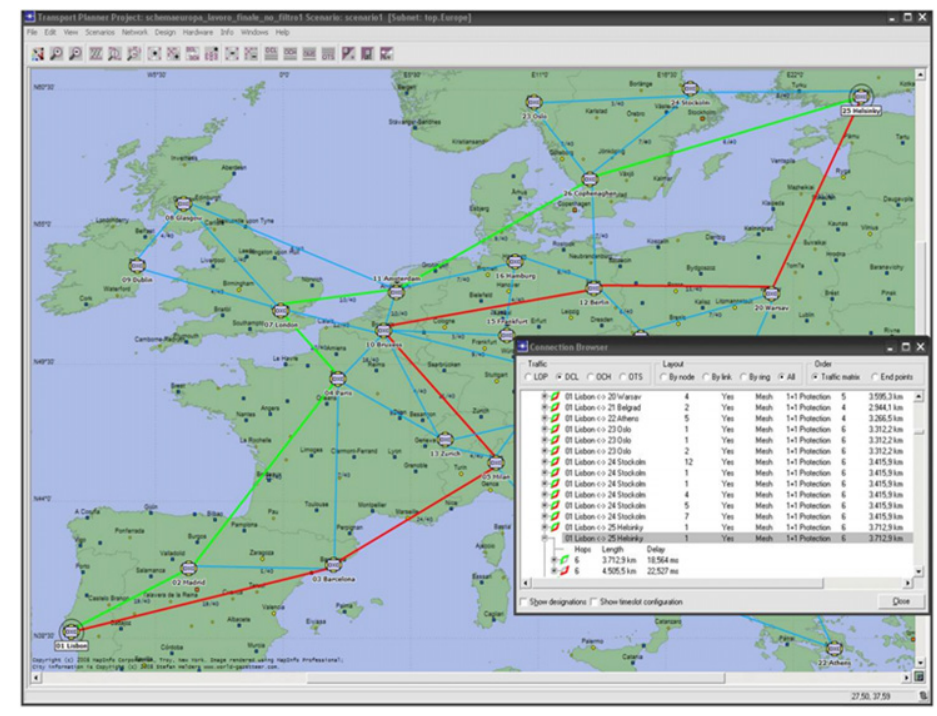

Fig. 4. Example of service and restoration paths between two nodes (Lisbon-Helsinki). 
Table 1

Wavelength assignment in the absence of OXC.

\begin{tabular}{|c|c|c|c|c|c|c|c|}
\hline & Link & Length (km) & Channel number & & Link & Length (km) & Channel number \\
\hline 1 & Lisbon-Madrid & 517.4 & 19 & 26 & Berlin-Hamburg & 257.4 & 6 \\
\hline 2 & Madrid-Barcelona & 521.7 & 6 & 27 & Berlin-Warsaw & 519.3 & 15 \\
\hline 3 & Barcelona-Lisbon & 1008.8 & 19 & 28 & Berlin-Copenhagen & 387.7 & 7 \\
\hline 4 & Barcelona-Paris & 829.4 & 7 & 29 & Zurich-Brussels & 523.6 & 4 \\
\hline 5 & Paris-Madrid & 983.0 & 21 & 30 & Munich-Milan & 462.5 & 5 \\
\hline 6 & Paris-Brussels & 259.9 & 16 & 31 & Munich-Brussels & 545.2 & 9 \\
\hline 7 & Paris-Zurich & 439.0 & 9 & 32 & Munich-Wien & 369.7 & 5 \\
\hline 8 & Milan-Barcelona & 725.1 & 24 & 33 & Frankfurt-Munich & 201.3 & 5 \\
\hline 9 & Milan-Rome & 483.3 & 10 & 34 & Hamburg-Munich & 331.5 & 4 \\
\hline 10 & Milan-Zurich & 198.3 & 4 & 35 & Prague-Berlin & 258.8 & 6 \\
\hline 11 & Rome-Munich & 827.9 & 6 & 36 & Prague-Warsaw & 437.1 & 7 \\
\hline 12 & Rome-Belgrade & 726.8 & 5 & 37 & Wien-Zurich & 735.9 & 9 \\
\hline 13 & London-Paris & 352.7 & 17 & 38 & Wien-Prague & 266.6 & 8 \\
\hline 14 & London-Dublin & 463.3 & 4 & 39 & Budapest-Prague & 433.8 & 3 \\
\hline 15 & London-Amsterdam & 351.9 & 10 & 40 & Budapest-Wien & 219.0 & 6 \\
\hline 16 & Glasgow-London & 552.1 & 3 & 41 & Warsaw-Budapest & 554.4 & 6 \\
\hline 17 & Dublin-Glasgow & 306.1 & 4 & 42 & Warsaw-Helsinki & 910.1 & 11 \\
\hline 18 & Brussels-Milan & 689.8 & 21 & 43 & Belgrade-Budapest & 308.9 & 7 \\
\hline 19 & Brussels-London & 323.0 & 12 & 44 & Athens-Rome & 1049.2 & 3 \\
\hline 20 & Brussels-Amsterdam & 175.4 & 10 & 45 & Athens-Belgrade & 809.4 & 3 \\
\hline 21 & Brussels-Berlin & 652.3 & 21 & 46 & Oslo-Copenhagen & 371.3 & 3 \\
\hline 22 & Brussels-Frankfurt & 374.3 & 3 & 47 & Stockholm-Oslo & 376.1 & 3 \\
\hline 23 & Amsterdam-Glasgow & 717.2 & 6 & 48 & Stockholm-Helsinki & 407.9 & 8 \\
\hline 24 & Amsterdam-Hamburg & 369.1 & 7 & 49 & Helsinki-Copenhagen & 771.9 & 6 \\
\hline 25 & Amsterdam-Copenhagen & 735.9 & 9 & 50 & Copenhagen-Stockholm & 474.9 & 7 \\
\hline
\end{tabular}
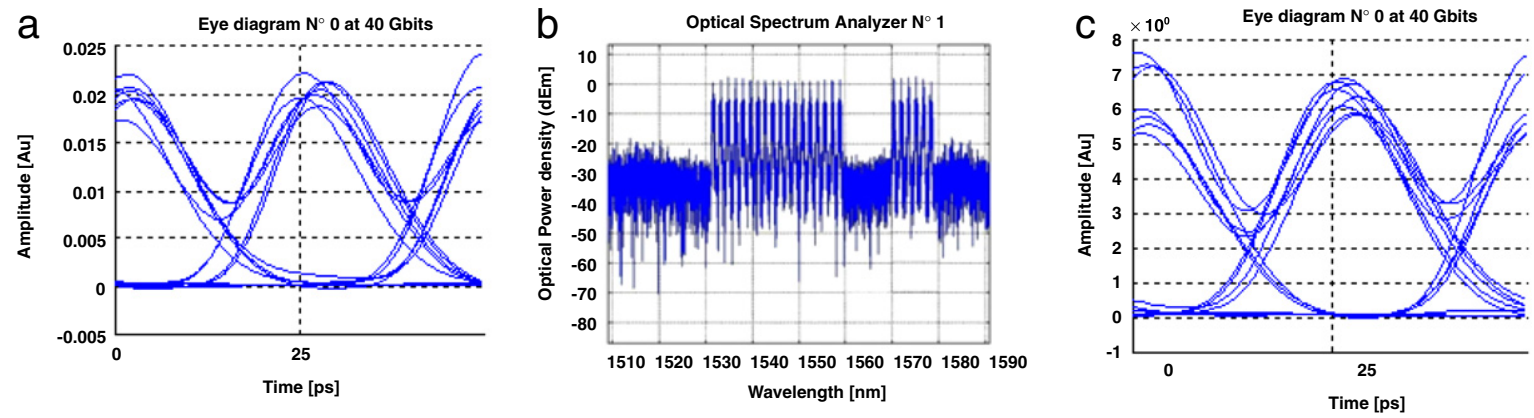

Fig. 5. Examples of $40 \mathrm{Gbit} / \mathrm{s}$ output transmission characteristics: (a) Eye diagram for $19 \times 40 \mathrm{Gbit} / \mathrm{s}$ dispersion management link measured on the 10 th channel after $1008 \mathrm{~km}$ (Lisbon-Barcelona): pulse duration $6 \mathrm{ps}$, peak power $32 \mathrm{~mW}$, frequency spacing $200 \mathrm{GHz}$. Corresponding $Q$ factor is 8.3 ; (b). Output spectrum of the $24 \times 40 \mathrm{Gbit} / \mathrm{s}$ dispersion management Milan-Barcelona link (725 km): pulse duration 6 ps, peak power 32 mW. Optical amplification occurs in band C and L; (c). Eye diagram of wavelength channel connecting Stockholm to Warsaw crossing OXCs in Copenhagen and Berlin, by adopting a pre-chirp with a pulse duration of 5 ps. Corresponding $Q$ factor is 8.6.

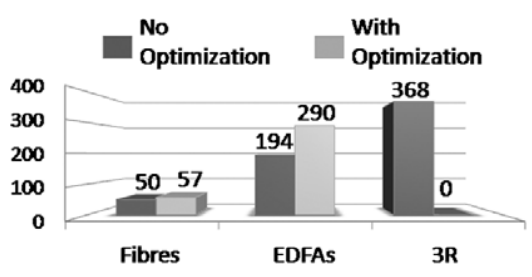

Fig. 6. Amplifiers and $3 R$ regenerators without and with optimization.

cases, we preferred to avoid the use of lightpaths requiring regeneration. Therefore, we took into consideration only 6 wavelengths connecting no-adjacent nodes. In Fig. 5(c), we report the eye diagram for the case of the wavelength connecting Stockholm to Warsaw (1380 km) crossing OXCs in Copenhagen and Berlin by adopting a pre-chirp (78 ps/nm/km) with a pulse duration of $5 \mathrm{ps}$.

\subsection{Results on the optimization of lightpaths considering traffic load variations}

In the presence of a traffic reduction, we can switch off links carrying less traffic, and we can re-route traffic in the wavelength channels operating in the remaining links. In Fig. 7, we report an example of switch off of links when the traffic reduces at $50 \%$ with respect to the one of Fig. 1 in the case Opaque Network: this operation can permit an energy saving of about $200 \mathrm{~kW}$.

As shown in Fig. 8, by reducing the traffic at $20 \%$ of Fig. 1 , 24 optical links can be switched off with an energy saving of about $300 \mathrm{~kW}$.

The energy saving could be much higher if we also consider the consumptions of other equipments as, for an instance, air-conditioning.

It has also to be pointed out that the links that have to be switched on in conditions of higher traffic can be 


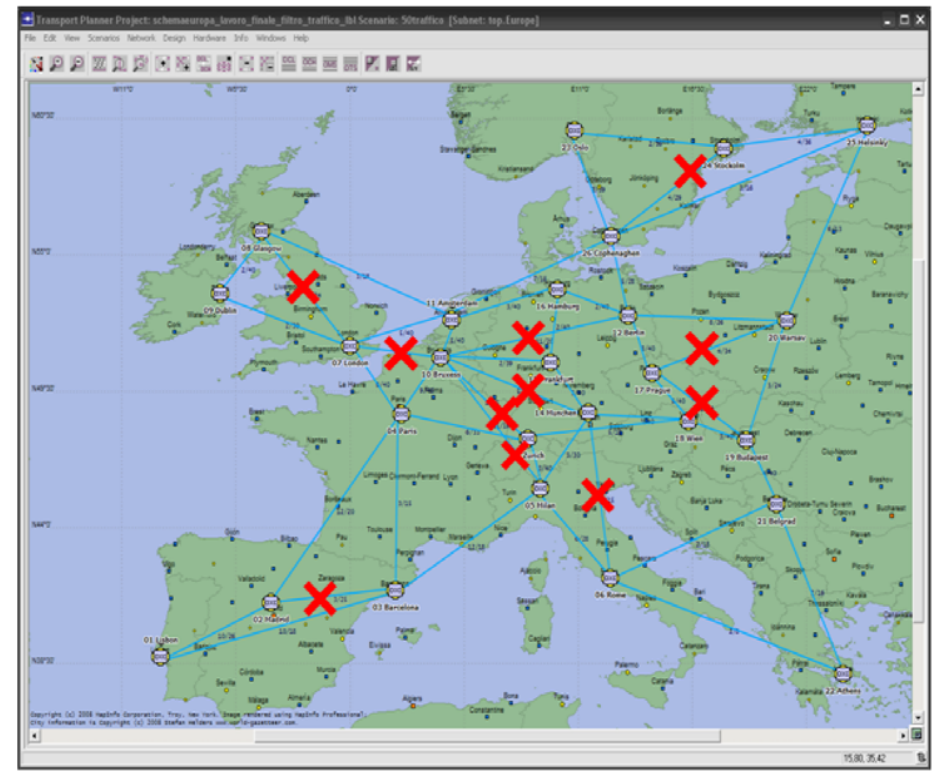

Fig. 7. Link switching off in the presence of a traffic reduction (50\% of Fig. 1).

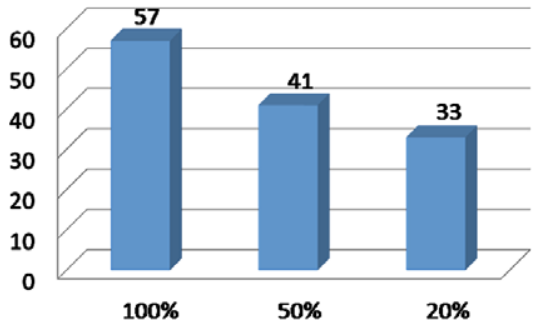

Fig. 8. Number of active link in the case of traffic reduction.

efficiently fed with solar cells, since the traffic load occurs mainly during the day time. For an instance, panels of some square meter can feed central for some kWp [5]. Therefore, a suitable project that takes into account both the reduction of transmission elements and the use of renewable energies, can give an important contribution in terms of no renewable energy saving.

\section{Wavelength assignment in a country network: Italy}

The role of Energy saving has been also investigated in a transport network with a smaller size, as for example the Italian transport network. In Fig. 9 we report the scheme of the Telecom Italia network [8]. In this case, due to traffic consideration, we assume the transmission based on $M \times$ $10 \mathrm{Gbit} / \mathrm{s} \mathrm{IM-DD} \mathrm{systems,} \mathrm{considering,} \mathrm{also} \mathrm{for} \mathrm{this} \mathrm{case,}$ G.652 fibres with dispersion management but without pre-chirp. It has to be pointed out that by adopting these transmission characteristics, no fundamental impairment is present in the links, since the maximum distance is equal to $370 \mathrm{~km}$; thus, no transmission optimization is needed, in particular $3 R$ devices are not required. Therefore, Energy saving can be achieved by optimizing the wavelength assignment and trying to limit the number of active links, and in particular trying to switch off the links carrying less traffic during the periods with less load.

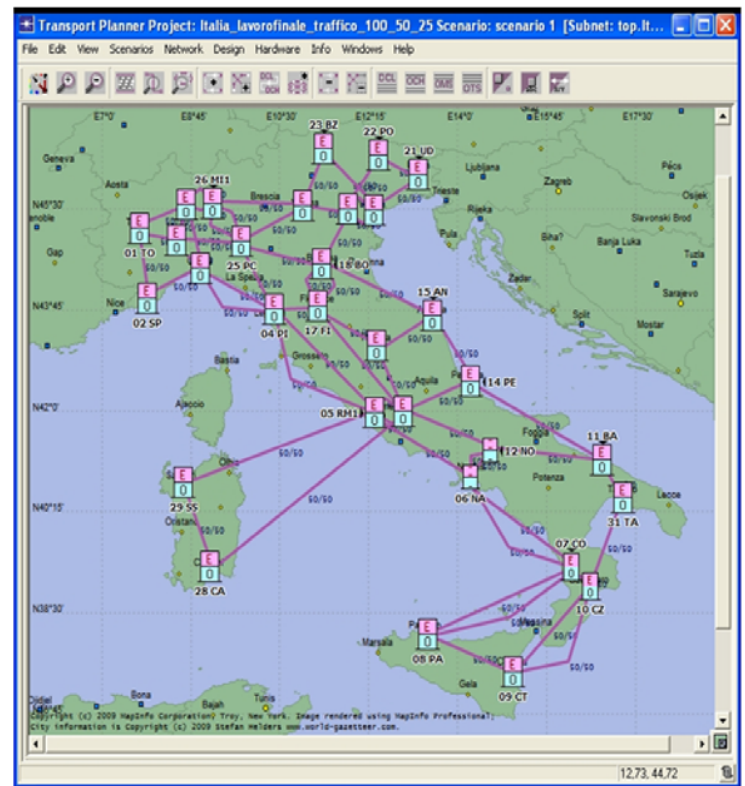

Fig. 9. A scheme of the Italian transport network based on the Telecom Italia network.

In Fig. 10 we report an example of traffic matrix for Italy in the case of maximum load considering 31 cities.

The network dimensioning is achieved by means of the OPNET code. In Fig. 11 we report the number of active links versus traffic load, and in particular we assume three cases: maximum load (100\%, corresponding to Fig. 10), medium load (50\% of the elements of Fig. 10) and low load (20\% of the elements of Fig. 10). As shown in Fig. 11, we can reduce the number of the active links up to $43 \%$, with an estimation of the energy consumption reduction equal to $200 \mathrm{~kW}$. Also in this case, it has to be pointed out that, due to the fact that the maximum load occurs 


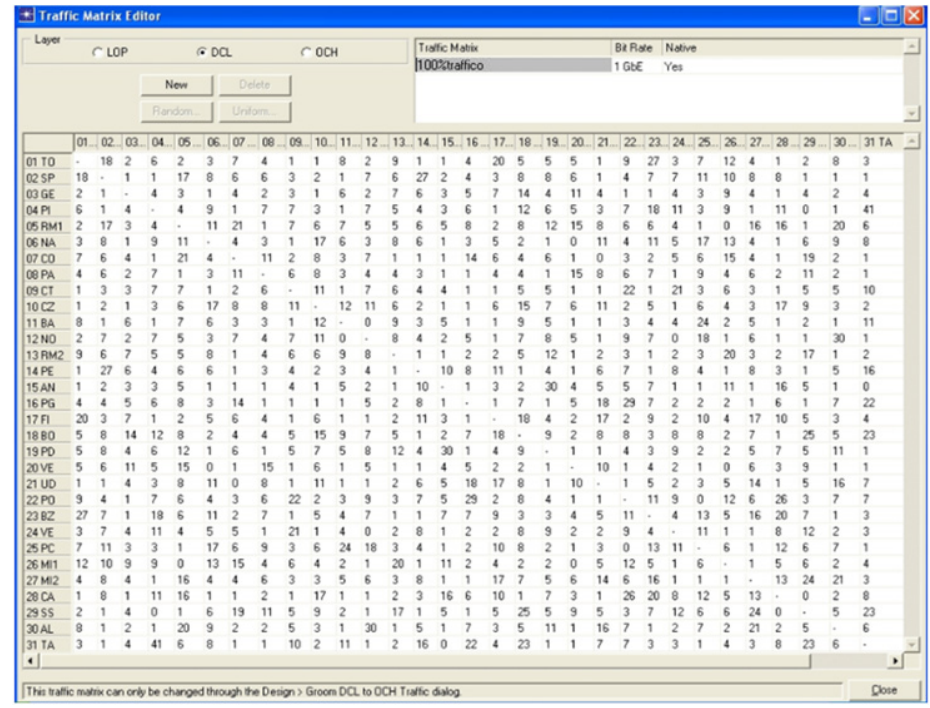

Fig. 10. Traffic matrix for Italy in conditions of maximum traffic.

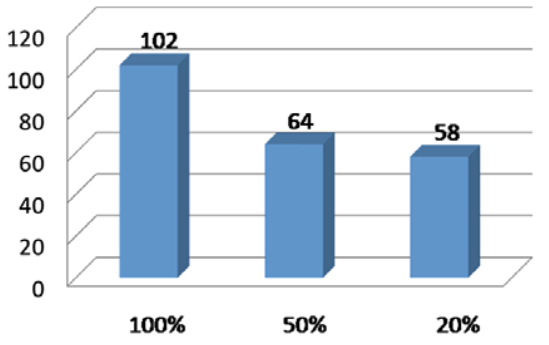

Fig. 11. Active links versus traffic load.

during the daytime, we could contribute to the apparatus alimentation by means of solar cells [9], especially for the in-line ones and the ones that can switched off during the night.

\section{Conclusions}

In this work we made an investigation on the optimization of the transmission resources in optical transport networks with the aim to achieve an energy saving. Such an investigation was based on two approaches: switching off of the links carrying less traffic in periods of low traffic load and optimization of the transmission performance. The first method is valid for any kind of transport network independently of its size, while the latter is important for wide area transport network where we can operate on the optimization of the transmission performance by using suitable transmission techniques, as the chromatic dispersion compensation, and optimizing the pulse duration, to avoid in-line $3 R$ regenerators. The behaviour of the traffic load suggest the use of solar energies to feed, also in partial way, the apparatuses that require to work during the daytime.

\section{Acknowledgements}

The work described in this paper was carried out with the support of the BONE project ("Building the Future Optical Network in Europe"), a Network of Excellence funded by the European Commission through the 7th ICTFramework Programme.

The authors thank OPNET for the license and the consultation on the OPNET simulation code.

\section{References}

[1] www.telecomitalia.it.

[2] J. Baliga, R. Ayre, V. Sorin, K. Hinton, R.S. Tucker, Energy consumption in access networks, in: Proc. of OFC/NFOEC 2008, San Diego, USA, paper OThT6.

[3] F. Matera, V. Eramo, A. Schiffini, M. Guglielmucci, M. Settembre, Numerical investigation on Design of Wide geographical Optical transport network based on $n \times 40 \mathrm{~Gb} / \mathrm{s}$ Transmission, J. Lightwave Technol. 21 (2003) 456-465.

[4] K. Ennser, R.I. Laming, M.N. Zervas, Analysis of 40Gb/s TDM transmission over embedded standard fiber employing chirped fiber grading dispersion compensators, J. Lightwave Technol. 16 (1998) 807-811.

[5] www.opnet.com.

[6] F. Matera, M. Settembre, Comparison of the performance of optically amplified transmission systems, J. Lightwave Technol. 14 (1996) $1-12$.

[7] M. Daikoku, I. Morita, H. Taga, H. Tanaka, T. Kawanishi, T. Sakamoto, T. Miyazaki, T. Fujita, $100 \mathrm{~Gb} / \mathrm{s}$ DQPSK transmission experiment without OTDM for 100G ethernet transport, J. Lightwave Technol. 25 (2007) 139-145.

[8] A. Allasia, V. Brizi, M. Potenza, Characteristics and trends of telecom italia transport networks, Fiber and Integrated Optics 27 (2008) 183-193.

[9] http://www.fvgenergy.com/photovoltaic_eng/home_eng.html. 